\title{
Mechanisms of apoptotic phosphatidylserine exposure
}

\author{
Cell Research (2013) 23:1247-1248. doi:10.1038/cr.2013.115; published online 27 August 2013
}

\begin{abstract}
It has been a long-standing enigma which scramblase causes phosphatidylserine residues to be exposed on the surface of apoptotic cells, thereby facilitating the phagocytic recognition, engulfment and destruction of apoptotic corpses. In a recent paper in Science, Nagata and coworkers reveal that the scramblases $\mathrm{Xkr8}$ and its $C$. elegans ortholog, CED-8, are activated by caspase cleavage in apoptotic cells.
\end{abstract}

All cells are separated from the extracellular environment by the plasma membrane, a phospholipid bilayer that prevents diffusion of proteins, ions and other essential molecules into the extracellular space and constitutes the structure in which membrane proteins are embedded. In animal cells, the lipid composition of the outer and inner leaflets of the plasma membrane is not symmetrical. Phosphatidylcholine (PC) and sphingomyelin (SM) are mainly present in the outer leaflet of the plasma membrane, whereas phosphatidylserine (PS), phosphatidylinositol (PI) and phosphatidylethanolamine (PE) are restricted to the inner leaflet. This lipid asymmetry is maintained by the combined action of ATP-dependent enzymes called flippases and floppases, which specifically translocate phospholipids and other molecules from the outer to the inner membrane leaflet and from the inner to the outer membrane leaflet, respectively [1]. Lipid composition asymmetry not only defines the curvature and electrochemical properties of the plasma membrane, but is also essential for the correct function of determined lipids, as for instance, PI, which only functions as a second messenger if present in the inner leaflet [2]. Nonetheless, several physiologically relevant processes as diverse as platelet activation, neurotransmitter release, sperm capacitation or apoptosis, require dissipation of plasma membrane lipid asymmetry, a process known as scrambling. The enzymes responsible for this activity are called scramblases, and function to randomize the distribution of phospholipids between both membrane leaflets in an ATP-independent manner [2-4].

Although plasma membrane asymmetry and the existence of flippases, floppases and scramblases have been known for decades, the identity of the specific enzymes involved in these activities has only begun to be revealed during the last few years. Very recently, the group of Shigekazu Nagata identified TMEM16F as the long sought-after calcium-dependent phospholipid scramblase [3]. However, to date, the identity of the scramblase(s) involved in apoptosis-related (and calcium-independent) PS exposure had remained elusive. Cell surface PS exposure is a classic feature of apoptotic cells and acts as an "eat me" signal allowing phagocytosis of postapoptotic bodies. In a recent paper in Science, Nagata's group identified XkRelated Protein 8 (Xkr8) as the enzyme responsible for this activity and demonstrated an evolutionarily conserved role of this protein in apoptosis-induced lipid scrambling [5].

To identify enzymes involved in membrane lipid scrambling, Nagata's group took advantage of their previously generated mouse $\mathrm{Ba} / \mathrm{F} 3$ pro- $\mathrm{B}$ cell line [3], which presented a high basal level of PS exposure. They then generated a cDNA library from $\mathrm{Ba} /$
F3 cells and overexpressed it in the parental cell line. Through sequential enrichment of cells with increased PS exposure, they were able to isolate a cDNA encoding the $\mathrm{Xkr} 8$ protein, which enhanced PS scrambling when overexpressed. Xkr8 overexpression (but not that of TMEM16F) was able to increase apoptosis-associated PS exposure. The authors then noticed that both impaired apoptosis-induced PS exposure and deficient post-apoptotic body clearance were correlated with low Xkr8 expression in leukemia and lymphoma cell lines, which was linked to hypermethylation of its promoter. Interestingly, these two alterations were reverted either by overexpressing $\mathrm{Xkr8}$ or by restitution of endogenous $\mathrm{Xkr8}$ expression after treatment with the demethylating agent 5-aza-2'-deoxycytidine (DAC), suggesting that methylation of the Xrk8 promoter may be a mechanism by which tumor cells evade their phagocytosis after apoptotic death, which may result in increased local inflammation, thus favoring tumor progression. Far from being restricted only to PS exposure, Xrk8 overexpression was able to promote scrambling of multiple lipid species during apoptosis, which was demonstrated by incorporation of fluorescent PC and SM analogues. This scrambling activity was restricted to apoptotic events, as Xkr8 overexpression had no effect on $\mathrm{Ca}^{2+}$-induced PS exposure. This specificity may be explained by the presence of an evolutionarily conserved caspase recognition site near Xkr8 C-terminal region, whose mutation prevented both Xkr8 cleavage by caspase- 3 or -7 and PS exposure during the course of apoptosis (Figure 1). These results 
from human cell lines were confirmed in $\mathrm{Xkr}^{-/-}$mouse embryonic fibroblasts and fetal thymocytes, which were unable to expose PS upon induction of apoptosis, underscoring the broad physiological relevance of Xkr8 in the apoptotic process. Finally, the authors moved to the nematode Caenorhabditis elegans to analyze whether the role of $\mathrm{Xpr} 8$ as lipid scramblase is evolutionarily conserved. C. elegans harbors only one ortholog of Xk proteins, CED-8, known to participate in the phagocytic removal of apoptotic corpses [6]. To determine the role of CED-8 in PS exposure, the authors took advantage of the "floater" assay, which is based on the appearance of floating cells ("floaters") that have detached from developing $C$. elegans embryos defective for apoptotic cell phagocytosis [7]. Nagata's group discovered that ced-8 deficiency leads to the accumulation of floaters. Moreover, ced-8 deficiency synergistically enhanced the number of floaters found in other engulfment mutants, which suggests that CED-8 function is not redundant to that developed by previously known engulfment mutants. This enhancing effect of ced-8 deletion was dependent on CED-3, the C. elegans ortholog of caspase-3, confirming the aforementioned results in mammalian cells. The authors then characterized that floaters resulting from $c e d-8$ deletion show a largely deficient PS exposure after developmental apoptosis, confirming the evolutionarily conserved role of Xk-related proteins in apoptosisinduced lipid scrambling. However, they observed that ced- 8 deletion does not lead to a total impairment in apoptotic PS presentation, suggesting that additional proteins must be involved in this process. Indeed, apoptosis-inducing factor can induce PS exposure in mammalian cells in a caspase-independent

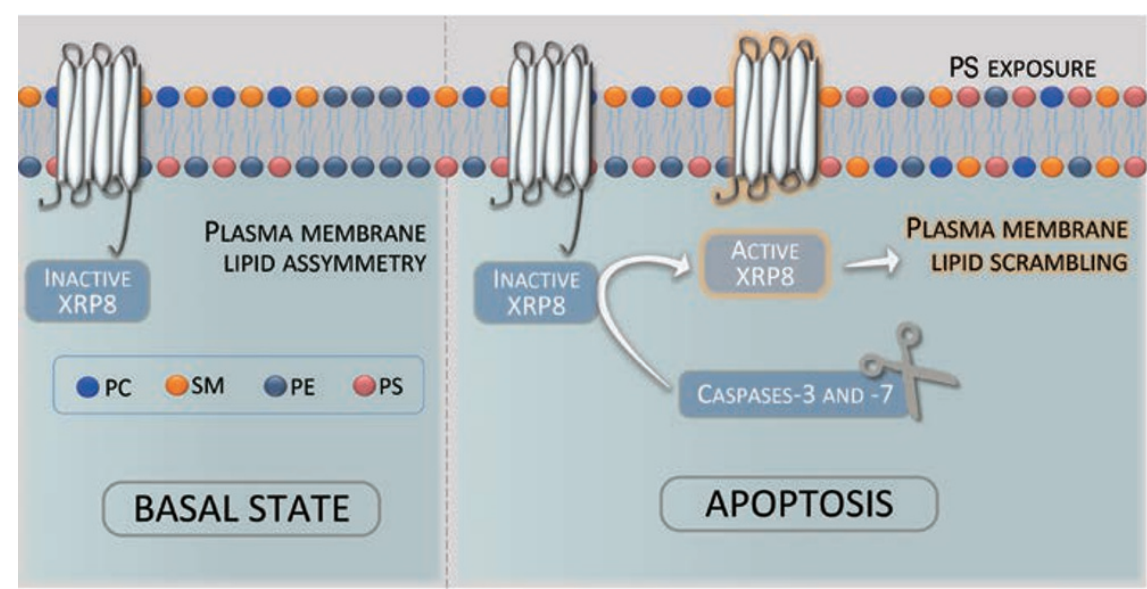

Figure 1 Xrp8 acts as apoptosis-induced lipid scramblase. Under normal conditions, the combined action of multiple mechanisms, including the activity of flippases and floppases, maintains lipid asymmetry between the outer and inner leaflets of the plasma membrane. Once apoptotic program is activated, caspases- 3 and -7 are able to cleave and activate Xrp8 protein, which acts as a lipid scramblase and leads to the loss of lipid asymmetry, resulting in PS exposure to the extracellular space. This acts as the "eat-me" signal that will allow phagocytosis of post-apoptotic cell corpses. PC, phosphatidylcholine; SM, sphingomyelin; PE, phosphatidylethanolamine; PS, phosphatidylserine.

fashion [8], and the C. elegans AIF ortholog, WAF-1, physically interacts with and activates another scramblase, SCRM-1 [4].

In summary, through a series of elegant manipulations, Nagata's group has found the long-sought caspaseactivated lipid scramblase that mediates the exposure of "eat-me" signals in post-apoptotic cell corpses. Further studies involving Xkr8 protein, including the mechanisms participating in its epigenetic repression may open new roads for the study of autoimmune diseases, such as lupus erythematosus, which is associated with failure in the post-apoptotic corpse clearance system.

\section{Guillermo Mariño $0^{1,2}$,} Guido Kroemer ${ }^{1,2,3,4,5}$

${ }^{1}$ INSERM, U848, F-94805 Villejuif, France; ${ }^{2}$ Université Paris Descartes/Paris V, Sorbonne Paris Cité, 75006 Paris, France, ${ }^{3}$ Equipe 11 la- bellisée par la Ligue Nationale contre le Cancer, Centre de Recherche des Cordeliers, F-75006 Paris, France; ${ }^{4}$ Pôle de Biologie, Hôpital Européen Georges Pompidou, AP-HP, F-75015 Paris, France; ${ }^{5}$ Metabolomics and Cell Biology Platforms, Institut Gustave Roussy, F-94805 Villejuif, France

Correspondence: Guido Kroemer

E-mail: kroemer@orange.fr

\section{References}

1 Leventis PA, Grinstein S. Annu Rev Biophys 2010; 39:407-427.

2 van Meer G, Voelker DR, Feigenson GW. Nat Rev Mol Cell Biol 2008; 9:112-124.

3 Suzuki J, Umeda M, Sims PJ, et al. Nature 2010; 468:834-838.

4 Wang X, Wang J, Gengyo-Ando K, et al. Nat Cell Biol 2007; 9:541-549.

5 Suzuki J, Denning DP, Imanishi E, et al. Science 2013; 341:403-406.

6 Stanfield GM, Horvitz HR. Mol Cell 2000; 5:423-433.

7 Denning DP, Hatch V, Horvitz HR. Nature 2012; 488:226-230.

8 Susin SA, Lorenzo HK, Zamzami N, et al. Nature 1999; 397:441-446. 